\title{
Application of Glass Material in Architectural Landscape Design
}

\author{
Hong Mao \\ Chongqing Vocational Institute of Engineering, Jiangjin 402260, China
}

Keywords: supervision and construction, glass material, application for architecture

\begin{abstract}
Glass is a special material made of silicon dioxide, which has been widely used in construction and mechanical production. The ancient artificial material, with its unique physical properties and potential artistic value, is increasingly being used in outdoor landscape. So far, glass has been extensively used in buildings as the landscape material such as the pavilion gallery painter, showing an irreplaceable art effect. However, glass material has not drawn enough attention in the current domestic landscape architecture. Thus, the paper will explore the comprehensive view of optics aesthetics and function in depth, and study on the specific application methods of glass in landscape construction.
\end{abstract}

\section{Introduction}

With the development of economy and the maturation of technology, more and more new materials have been applied to landscape architecture, and glass material is one of them. Because of the main components of the glass is silicon dioxide structure is relatively stable, so the glass material has a good durability and corrosion resistance, but also has both good decorative, completely can be used in landscape architecture design and construction. Therefore, we need to use glass materials flexibly to create better landscape architecture.

Glass has been widely used in interior and architecture because of its cold and transparent physical properties. However, at present, the application of glass materials in China is not mature, and lacks perfect theoretical system and technical guidance. With the continuous improvement of technical level, the application of glass materials has gained more and more attention.

\section{Concrete analysis of glass material in existing technology}

First of all, the glass material has transparency which can be used for the perspective of scenery, and can meet people's visual needs while narrowing the space distance. Secondly, mirror glass has good emission properties, which can reflect the images of surrounding scenery on the mirror to produce better landscape effect. Third, through the processing of glass, we can get more colorful glass, and make up for the lack of color of traditional landscape materials. Fourthly, because of the special nature of glass, glass can be processed into many different shapes by means of melting die-casting and blowing. Fifth, there is a better effect on heat insulation sound insulation and noise reduction; finally, glass is a recyclable material, through the use of glass, can achieve the sustainable development of building materials.

Glass material defects of course, glass materials are not perfect, but also have certain defects. First of all, the bending strength and flexural strength of glass are lower, and the resistance to impact is poor. When the external load exceeds the capacity of the material itself, the rupture and damage will occur. In addition, the thermal stability of the glass material is poor, and in the case of temperature change, the physical capacity of the glass will drop sharply and even crack or crack. Therefore, we need to further solve the problems of glass in performance through post-processing.

Material such as glass is much less than the brick that is widely application in landscape design, but the glass material's unique charm deeply attracted by the people, the scope of its application in landscape design is more and more extensive, and often become the most attractive part of landscape 
design.

\section{Concrete application of glass in landscape construction}

For the practical application of glass material in landscape, we can analyze the application of optical Angle mechanics Angle and other aspects.

The first is transparency. As the basic characteristic of glass, transparency is the biggest characteristic of glass, which is often applied in the construction of landscape architecture. By applying the transparent characteristic, can further promote the mixing and infiltration of indoor and outdoor landscape, at the same time can be further help bridge the gap space feeling, further close the distance between human and nature, let people can appreciate the natural scenery more directly.

According to existing optical research, the optical utilization of glass material can be shown in two aspects. One is secular reflection, the other is diffuse reflection.

The first thing we're going to talk about is secular reflection, and by coating the back of the glass with a sheet of metal, the mirror effect is completely reflected. With the further processing of this mirror glass, we can get a variety of forms conducive to the landscape, which can create a more comprehensive and richer view of the landscape. Such as glass curtain wall of the popular, is the use of the effect of total reflection mirror glass, use of limited space, create the unlimited sense on the vision, enhance the people appreciate the landscape of interesting experience.

In addition to the secular emission, what we need to know is diffuse reflection. Through the use of technology such as frosting and sandblasting to transform the glass, the smooth and transparent glass has a soft light effect between the light and the opaque light. It also reflects some of the light while shading it. Use of common glass, sand and sand blasting process in the grinding of machining, surface roughness, at the same time, depending on the degree of roughness, can make glass with light transmittance of different level. Using diffuse reflection glass, it can make the glass in diffuse light and shadow environment, forming a unique soft landscape effect.

Of course, we can also make the glass light and light transmittance soft, and get a visual double effect. The most direct way is to make the landscape lamp. Combined with lighting fixtures, we can use the optical properties of glass to produce the effect we need. In the actual view, we can choose glass as landscape lamp, used to create a good atmosphere at night, with the artists create graphic patterns, can have the dual effect of landscape lighting and performance. For example, the LED lamp is used to cooperate with the ground glass, which can form a linear lamp belt, and when the ground application lamp belt is made, the frosted glass can also play an anti-skid role and improve the safety factor.

In addition, we can also use glass Mosaic and LED lights for organic union, in the way of, constitute a different color and different shape, which can express different landscape.

\section{Mechanical application of glass materials}

In addition to optical properties, glass material has many mechanical properties. They can be used as ceiling, guardrail, and ground material. In the application of mechanical properties, glass can be divided into plane glass and deep processing glass. Among them, the mechanical parameters of flat glass are often as the change of raw material quality production technology level and changes, and deep processing of glass has some common glass do not have special properties.

Safety glass is a product of deep glass processing. Through deep processing, the safety glass has higher mechanical strength and is more prominent in the impact resistance. At present common safety glass mainly has toughened glass laminated glass and wired glass.

The first is tempered glass. In the process of casting, an effective pressure layer is formed on the glass surface through physical or chemical methods. Glass has high compressive strength and compressive strength. In the case of external forces, the tempered glass can be partially offset by the pressure layer, thereby avoiding the rupture or damage of the glass because of the external force. In the actual construction process, the tempered glass is usually applied to the barrier and the ground. 
Some mountain scenic area, for example, toughened glass is often used in glass covered Bridges, in terms of its physical properties, toughened glass itself weight is lighter, but the stress coefficient of physical parameters is higher, so better suited to be used in class of bridge landscape construction, at the same time the bridge gallery design can bring visitors better sensory enjoyment, pull close the distance people and scenery, at the same time satisfy the visitors' curiosity and adventure.

Next, let's talk about the laminated glass. So-called sandwich glass, just as its name implies is like a sandwich biscuit between the double layer or multilayer glass clamp or other substances, such as rubber, membrane and after pressing forming the integration of the complete glass, in the landscape construction of the actual, we can according to the specific needs, add glue in glass sandwich class material, or silk screen and the printing pictures, etc. This kind of glass not only has very strong stress resistance, but also the safety factor is very high, even if broken apart also won't hurt the person.

Wired glass is a kind of laminated glass; laminated glass for this usually will be treated with preheating wire through the special craft of pressing pressure into the glass in the middle, after annealing process of special laminated glass cutting processing. Particularly high stress coefficient of resistance of the glass, is usually applied in a large area of light inlet window is made, at the same time, in the process of design and production of concrete will be fully integrated beautiful, lighting, cleaning, cooling factors into the design idea, can let the wired glass play better results.

In the concrete landscape construction, the laminated glass is often applied to the ceiling design of the pavilion. There is a high frequency of use in the building of the bus stop park feature pavilion. At the same time, in the building of the mall large public places, the laminated glass also has a wide application space.

Decorative glass, usually to cut the original glass coloring and other physical processing form a new type of glass, in the specific process of landscape space, decorative glass will create a more colorful landscape atmosphere, rise to decorate the facade shape the landscape of the specific function.

In China, glass has a long history. For example, in the construction of lignin garden 200 years ago, this kind of glass has already been used. In this way, through the special material composition framework, after the Mosaic of the glass, through etching processing, into glass etching processing art. In the current production of glass art, etching is a special form. Imported glass material, using new type of etching processing technology, fully fusion of Chinese and western cultural characteristics, such as red, yellow, green and purple in color as the primary colors, with different characteristics of lattice design, make the window has a unique and elegant artistic breath, and Chinese classical garden art supplement each other.

Through different processing methods, we can make the glass from the original colorless transparent to colorful. Usually, the dyeing process of glass can be divided into coloring coating color painting and so on. Through the processing of color, we can obtain the effect of different landscape atmosphere. During the design process, the designer will bring the sensory shock to people according to the color change of art psychology. Through the processing of the color of the landscape, the landscape can show the psychological sensation of cold and warm contraction and expansion. Through the color building atmosphere, the color enhancement space, the combination of light and color, we can create a more perfect artistic landscape.

Similar to the ceramic burning, the glass landscape can also be used to achieve the desired artistic effect. The inorganic glaze is attached to the glass, and the glaze is completely sintered on the surface of the glass. Normally, just have glass can be applied to the design of sound barriers and other specific, on the one hand, can enhance the ability of wall sound insulation noise, on the other hand also can improve the traditional wall art effect, bring one bright spot for dark side walls.

With unique physical properties, glass can be matched with other materials to achieve better artistic effects. In landscape construction, different artistic effects can be achieved by combining glass with metal, stone, wood and concrete. Combined with metal, for example, can represent lightsome glass at the same time, more prominent metal itself has strong rigid solid feeling, at present, through the modular design, to the fabrication of glass and other materials, can be further bring landscape design features of beauty of science and technology. 


\section{Conclusions}

The garden is composed of natural scenery, because of its unique artistic atmosphere, gradually evolved into a landscape culture. Generally speaking, the landscape itself cannot be displayed, and they often need to be shown through a certain medium. Material is the basis of the landscape, as if the air is too human, building materials is the development of the natural landscape is one of the most basic, and its artistic expression, it is through the material to display. Different design style requires different material, is closely linked between them, and not only so, material but also the foundation of design, without the proper materials, then design style can reflect. The landscape design also needs to be innovated, and the innovation here contains two meanings: first, the development of new building materials; In addition, the original building materials have been developed into new functions, and all things in the world have their own unique properties, and building materials are no exception. The structure inside the glass is quite complex, which can be said to be infinitely changeable, and with this change, their function and form have also changed. To a certain extent, a material's aesthetic, is determined by physical properties of the material, so it requires the staff to have a detailed knowledge of the glass, so to their form and function.

Art comes from life. In the landscape design and construction, the artistic expression and artistic value of glass are self-evident. Combined with the current development of technology, the use of glass space will be more and more wide, will bring new breakthrough for our landscape construction, and in the scientific, driven by glass landscape will have more stronger function. We hope that the glass material will be more outstanding in the future landscape construction work.

\section{References}

[1] The application of ferrogenic glass materials in landscape design in the year of he xue. Chinese Garden, 2015, 12: 120-124. (In Chinese)

[2] Tan fang liang's application of glass materials in landscape materials. Modern Gardening, 2011, 07: 79. (In Chinese)

[3] Lin Z. Inheritance and Innovation of Culture in Contemporary Architectural Landscape Design. Journal of Anhui Agricultural Sciences, 2009: 36-41.

[4] Lian Y, Cai J. Application of Fengshui Doctrines in Landscape Architecture Design. Journal of Landscape Research, 2012, (8): 56-64.

[5] Yun M. The Study on Application of Natural Materials in Landscape Architecture Design Based on the Experimental Simulation. International Conference on Intelligent Computation Technology and Automation. IEEE, 2015: 857-860.

[6] Li Z, Wang M. The application of Alar culture on architectural landscape design. Shanxi Architecture, 2017, 9(3): 79-86.

[7] Xu H, University A J. On the Application of Traditional Chinese Architectural Symbols of Jiangnan Areas in the Landscape Design. Journal of Heihe University, 2017, 8(1): 53-59.

[8] Zhang L, You Z. Application of Confucian Cultural Concepts in the Landscape Design of Chinese Architecture. International Conference on Education, Sports, Arts and Management Engineering. 2017, 6(5): 45-56.

[9] Fielding H, Lamoine G. Application of Digital Technology in Landscape Architecture Design. Modern Agricultural Sciences \& Technology, 2010, 56(4):696-703. 